\title{
Neuroendocrine liver metastases: a contemporary review of treatment strategies
}

\author{
Jordan M. Cloyd ${ }^{1}$, Aslam Ejaz ${ }^{1}$, Bhavana Konda ${ }^{2}$, Mina S. Makary ${ }^{3}$, Timothy M. Pawlik ${ }^{1}$ \\ ${ }^{1}$ Departments of Surgery, ${ }^{2}$ Departments of Internal Medicine, ${ }^{3}$ Departments of Radiology, The Ohio State University, Wexner Medical Center, \\ Columbus, OH, USA \\ Contributions: (I) Conception and design: JM Cloyd, TM Pawlik; (II) Administrative support: TM Pawlik; (III) Provision of study materials or \\ patients: JM Cloyd, TM Pawlik; (IV) Collection and assembly of data: JM Cloyd; (V) Data analysis and interpretation: All authors; (VI) Manuscript \\ writing: All authors; (VII) Final approval of manuscript: All authors. \\ Correspondence to: Timothy M. Pawlik, MD, MPH, PhD, FACS, FRACS (Hon). Professor and Chair of Surgery, The Urban Meyer III and Shelley \\ Meyer Chair for Cancer Research, Professor of Surgery, Oncology, Health Services Management and Policy, Department of Surgery, The Ohio \\ State University, Wexner Medical Center, 395 W. 12th Ave., Suite 670, Columbus, OH, USA. Email: Tim.Pawlik@osumc.edu.
}

\begin{abstract}
Well-differentiated neuroendocrine tumors (NETs) are globally increasing in prevalence and the liver is the most common site of metastasis. Neuroendocrine liver metastases (NELM) are heterogeneous in clinical presentation and prognosis. Fortunately, recent advances in diagnostic techniques and therapeutic strategies have improved the multidisciplinary management of this challenging condition. When feasible, surgical resection of NELM offers the best long-term outcomes. General indications for hepatic resection include performance status acceptable for major liver surgery, grade 1 or 2 tumors, absence of extrahepatic disease, adequate size and function of future liver remnant, and feasibility of resecting $>90 \%$ of metastases. Adjunct therapies including concomitant liver ablation are generally safe when used appropriately and may expand the number of patients eligible for surgery. Among patients with synchronous resectable NELM, resection of the primary either in a staged or combined fashion is recommended. For patients who are not surgical candidates, liver-directed therapies such as transarterial embolization, chemoembolization, and radioembolization can provide locoregional control and improve symptoms of carcinoid syndrome. Multiple systemic therapy options also exist for patients with advanced or progressive disease. Ongoing research efforts are needed to identify novel biomarkers that will define the optimal indications for and sequencing of treatments to be delivered in a personalized fashion.
\end{abstract}

Keywords: Gastroenteropancreatic neuroendocrine tumors (GEP-NETs); liver-directed therapies; carcinoid; hepatic resection; targeted therapy

Submitted Feb 17, 2020. Accepted for publication Mar 31, 2020.

doi: $10.21037 / \mathrm{hbsn} .2020 .04 .02$

View this article at: http://dx.doi.org/10.21037/hbsn.2020.04.02

\section{Introduction}

Neuroendocrine tumors (NETs) are a rare but increasingly prevalent neoplasm with heterogeneous clinical behavior. Well-differentiated gastroenteropancreatic (GEP) NETs are typically characterized by an indolent clinical course and relatively good prognosis compared with other gastrointestinal cancers. In contrast, GEP-NETs frequently metastasize to the liver with approximately $28-77 \%$ of patients developing neuroendocrine liver metastases (NELM) in their lifetime $(1,2)$. NELM can be associated with significant detriments in quality and quantity of life (3). Indeed, the presence of NELM is one of the most significant negative prognostic factors for long-term survival among patients with NETs (4). In addition, patients with liver metastases can experience debilitating carcinoid syndrome, as well as local complications such as biliary obstruction or liver insufficiency $(5,6)$. For these reasons, 
management of NELM is important as liver disease is often the main determinant of patient prognosis among individuals with otherwise indolent disease. Fortunately, significant advances in novel imaging techniques, liverdirected therapies, and systemic treatment options have improved the multidisciplinary management of patients with NELM $(3,7,8)$. We herein review recent advances, as well as provide a contemporary overview of the diagnosis and management of patients with well-differentiated NELM.

\section{Diagnosis}

\section{Initial diagnosis}

All patients with a diagnosis of NET and concern for possible NELM should undergo comprehensive history, physical, biochemical and radiographic evaluation. The history should focus on previous diagnostic procedures and treatments received. Symptoms consistent with carcinoid syndrome and/or functional tumor syndromes should be carefully elicited. The physical examination should be comprehensive and include signs and symptoms of chronic liver disease. Standard laboratory tests including complete blood count, prothrombin time, and liver function tests provide evidence of liver synthetic function and will inform patient eligibility for liver-directed therapy. Tumor markers including chromogranin A, synaptophysin, pancreatic polypeptide, gastrin, and insulin should be ordered as these levels may be useful to measure tumor response to treatment and/or recurrence. For individuals in which the presence of carcinoid syndrome is indeterminate, 24-hour urinary serotonin can be measured.

While a biopsy is not required to document NELM in a patient with a history of GEP-NETs and liver lesions with classic radiographic features, histologic confirmation can be helpful in a patient where the diagnosis is unclear. Biopsy can be performed percutaneously or at the time of surgery for some other indication (e.g., resection of the primary or cholecystectomy); less commonly, endoscopic ultrasound can be used to biopsy lesions in the left liver. Careful attention to the differentiation, overall grade, and $\mathrm{Ki}-67$ are paramount as this information is both prognostic and may inform treatment decision-making. While most primary and metastatic NETs are concordant, occasionally the Ki-67 rate of the NELM may be different than the primary tumor (9).

\section{Imaging}

Patients with NETs should undergo triple-phase (noncontrast, arterial phase, and portal venous phase imaging) computed tomography (CT) of the chest, abdomen, and pelvis. NELM are typically hyperenhancing on arterial phase with persistent enhancement on portal venous phase, though enhancement patterns can vary (10) (Figure 1). Large tumors can exhibit central areas of necrosis. In general, magnetic resonance imaging (MRI) is excellent for characterizing liver lesions. While enhancement patterns can vary, NELM are typically best visualized on T1 hepatic arterial and T2 fat-suppressed fast spin-echo phases on conventional MRI. More recently, diffusionweighted MRI has shown improved detection for NELM $(11,12)$.

Somatostatin receptor scintigraphy (SRS) also plays an important role in the radiographic staging of patients with suspected NELM given that over $90 \%$ of NETs express somatostatin receptors. While traditionally the octapeptide analog of somatostatin (octreotide) labeled with indium-111 (known as OctreoScan) was the standard method of staging and characterizing NETs, this has largely been replaced at most centers by gallium ${ }^{68} \mathrm{Ga}$ DOTATATE positron emission tomography (PET). Previous studies have suggested that the routine incorporation of ${ }^{68} \mathrm{Ga}$ DOTATATE may alter treatment decision making in up to one-third of cases and provides important prognostic information $(13,14)$. Based on its increased sensitivity, reduced radiation dose, improved registration with $\mathrm{CT}$, and reduced scanning times required (15), consensus guidelines have recommended that SRS-PET should replace OctreoScan for all indications in which the latter was previously used (16) (Figure 2).

Accurate preoperative imaging is essential for assessing resectability and informing liver-directed therapies. A recent review found pooled sensitivity rates of $22 \%, 21.2 \%$, $32.6 \%$, and $12.4 \%$, respectively for detecting NELM by ultrasound, CT, MRI, and SRS, respectively while accuracy was reported to be $38.4 \%, 37.6 \%, 48.8 \%$, and $23.9 \%$, respectively (17). The use of contemporary ${ }^{68} \mathrm{Ga}-$ somatostatin receptor PET/CT has improved sensitivity and specificity for NELM to approximately $94 \%$ and $89 \%$, respectively (17). In practice, CT, MRI, and SRS-PET are all useful in the evaluation of patients with metastatic NET, particularly those who are being considered for hepatic resection, and are supported by recent consensus guidelines 

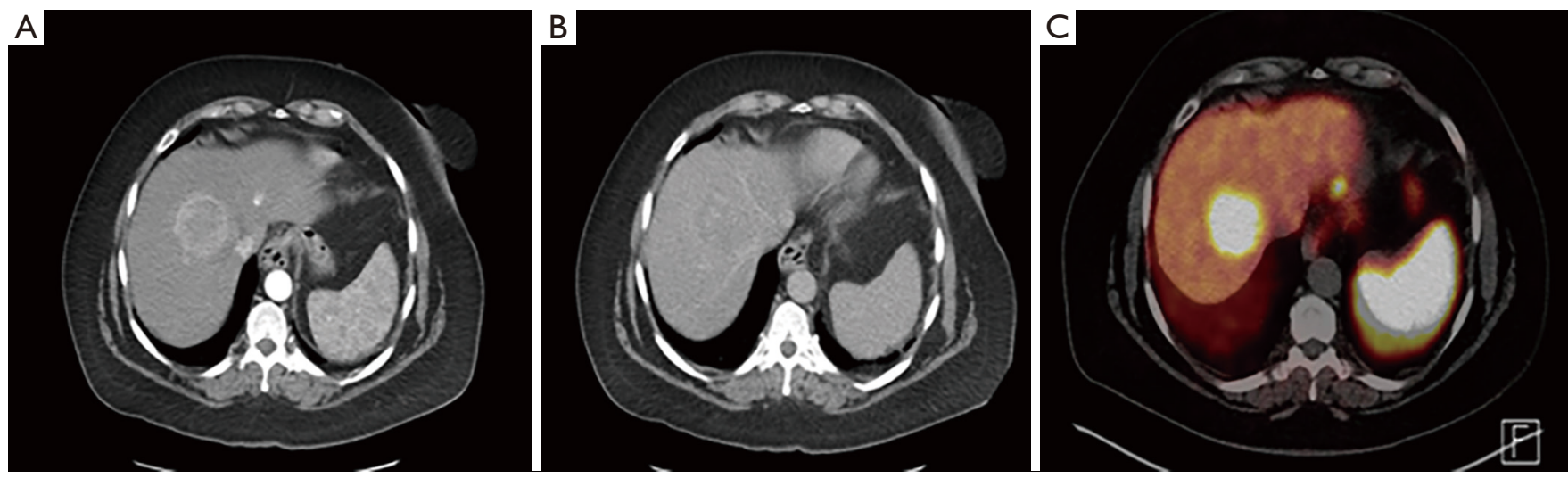

Figure 1 Representative example of NELM on (A) arterial phase CT, (B) portal venous phase CT, and (C) ${ }^{68}$ Ga-DOTATATE CT-PET. NELM, neuroendocrine liver metastases; CT, computed tomography; PET, positron emission tomography.

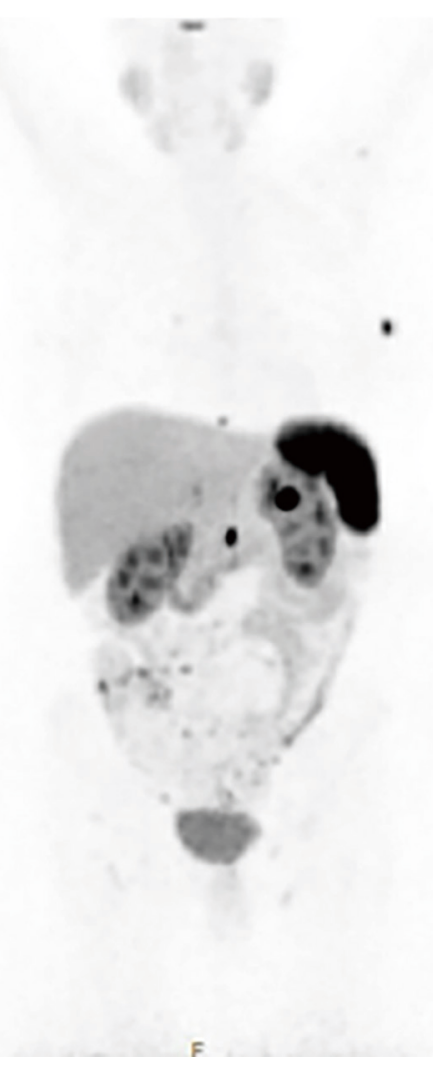

Figure 2 Representative example of ${ }^{68}$ Ga-DOTATATE CT-PET demonstrating somatostatin receptor avid metastatic disease in the breast, peritoneum, bone, and lymph nodes in a patient with unremarkable CT imaging. CT, computed tomography; PET, positron emission tomography. from the European Neuroendocrine Tumor Society (ENETS) (18).

\section{Role of hepatic resection}

\section{Outcomes}

Although no randomized controlled trials have been performed comparing surgery versus non-surgical therapies (19), hepatic resection of NELM, when feasible, is thought to be associated with the best long-term outcomes. In fact, 5-year overall survival (OS) can reach 60-80\% among well-selected patients (20). Several metaanalyses have attempted to confirm the benefit of surgery for NELM. For example, a 2012 systematic review reported pooled median 3-, 5- and 10-year OS of $83 \%$ (range, 63-100\%), 70.5\% (range, 31-100\%), and 42\% (range, $0-100 \%)$, respectively (21). The survival benefit associated with hepatic resection persisted even compared with patients who were treated with aggressive nonoperative approaches. For example, a retrospective multi-institutional review of 339 patients with NELM undergoing liver resection versus 414 patients receiving intra-arterial therapies noted an improved 5-year OS among patients who underwent surgery (74\% vs. $30 \%, \mathrm{P}<0.001)$ (22). Another systematic review noted significantly improved survival outcomes following liver resection versus patients who received liverdirected therapies, chemotherapy, or no treatment (23).

Since a prospective randomized controlled trial is 
unlikely to be performed, and given the excellent longterm outcomes observed in retrospective series of patients undergoing resection for NELM, hepatic resection is recommended for appropriately selected patients. A recent international Working Group on Neuroendocrine Liver Metastases similarly concluded that the use of liver resection is the preferred treatment option for patients with NELM (3). Similar guideline recommendations have been made by the North American Neuroendocrine Tumor Society (NANETS) (24) and ENETS (1), as well as other organizations (25-27).

\section{Prognostic factors}

A better understanding of relevant prognostic factors may improve patient selection for liver resection. Multiple studies have been conducted to evaluate risk factors for long-term outcomes following hepatic resection of NELM. For example, Glazer et al. reported that synchronous disease, nonfunctional hormonal status, and extra-hepatic disease were associated with worse long-term outcomes, while Spolverato et al. reported that extrahepatic disease and tumor grade were adversely associated with prognosis $(28,29)$. In a systematic review, Saxena et al. noted that poor histologic grade, extrahepatic disease, and a macroscopically incomplete resection were negative prognostic factors (21). While extrahepatic metastatic disease is one of the most consistent prognostic factors observed across studies, hepatic resection in the setting of low-volume indolent bone metastases may be appropriate (30). In addition, synchronous disease is associated with higher recurrence rates compared with metachronous disease (31). Interestingly, most, but not all studies, have noted that $\mathrm{R} 1$ margin status is not associated with worse outcomes (29,32-34).

\section{Debulking}

The role of debulking surgery for NELM continues to be debated with many surgeons advocating for aggressive surgical intervention even when complete macroscopic removal of all disease is not possible (35). Several series have reported excellent long-term outcomes and symptom control among patients undergoing cytoreductive surgery with $80-90 \%$ of NELM tumors removed. For example, Ejaz et al. reported a 5-year OS of $60 \%$ among 179 patients with NELM who underwent non-curative cytoreductive surgery (36). In a separate study, Sarmiento et al. reported a 5 -year OS of $61 \%$, in addition to excellent symptom control among 170 patients who underwent $90 \%$ debulking of NELM (37). Increasingly, some surgeons have recommended consideration of debulking surgery even when less than $90 \%$ of disease can be removed. For example, Scott et al. demonstrated improved OS among patients who had $>70 \%$ of metastatic disease resected (38), while Morgan et al. noted comparable survival outcomes for patients who had $70 \%, 90 \%$, and $100 \%$ of tumor volume resected (35). These studies are difficult to interpret given the inherent selection biases present in institutional retrospective studies; however based on these data, some investigators have proposed changing the threshold for considering cytoreductive surgery to a 70\% threshold (35).

\section{Concomitant ablation}

Ablative procedures are commonly used for oligometastatic disease either alone or in conjunction with hepatic resection. Concomitant ablation is mainly used in the setting of multifocal disease to assist with debulking of unresectable disease or to avoid extended liver resection. Contemporary studies appear to demonstrate favorable long-term outcomes associated with this strategy. For example, Taner et al. reported that ablation used in combination with resection resulted in 5- and 10-year OS of $80 \%$ and $59 \%$, respectively (39). In a large multiinstitutional analysis, ablative procedures were used in approximately $20 \%$ of patients undergoing surgery for NELM, which was not associated with an increased risk of recurrence or worse survival (40). In addition, concomitant ablation did not appear to increase the perioperative risks of surgery for NELM (41). In general, ablation should be used for small-intermediate sized, centrally-located tumors to preserve liver parenchyma or avoid the morbidity of a major resection. Care should be taken when combining ablation with extended hepatectomy as injury to the future liver remnant could increase the risk for postoperative hepatic insufficiency (42). Finally, tumors close to the hepatic veins (heat sink) or portal pedicles (biliary injury, liver ischemia) may be at higher risk of incomplete ablation or complications, respectively.

\section{Recurrent disease}

While long-term OS generally is excellent, recurrence following hepatic resection of NELM can approach $50-95 \%$, and the majority of recurrences will occur in the 
liver $(21,40)$. Among patients who develop intrahepatic recurrence, $>70 \%$ will occur within 3 years of surgery, and approximately $40 \%$ within 1 year $(34,43)$. Multiple studies have highlighted the safety and relative efficacy of repeat hepatectomy for recurrent primary and metastatic liver disease $(44,45)$; repeat hepatic resection for recurrent NELM has similarly been demonstrated to be feasible and associated with good long-term survival outcomes in wellselected patients (46). For patients with recurrent NELM who are not surgical candidates, non-surgical therapies are alternative options.

\section{Surgical considerations}

\section{Patient selection}

Careful patient selection is imperative for enhancing perioperative and long-term outcomes associated with hepatic resection for NELM. In general, the liver metastases should be amenable to complete or $>90 \%$ resection. Hepatic resection should leave a future liver remnant with at least two contiguous segments characterized by intact venous, arterial, portal venous, and biliary drainage that comprises at least $20 \%$ of standardized liver volume. Minimal to no extrahepatic disease should be present and, in general, the primary tumor should be resectable. Hepatic resection is generally indicated only for tumors with welldifferentiated morphology and low-intermediate grade though limited evidence suggests acceptable outcomes in high-grade tumors particularly when the Ki-67 <55\% (47). Patients should be healthy enough to undergo major hepatic resection with acceptable morbidity. For patients with prohibitive or borderline comorbidities who cannot be optimized, other liver-directed therapies should be prioritized. ENETS has previously published guidelines on indications for hepatic resection of NELM: R0 resection feasible, grade 1 or 2 tumors, acceptable perioperative morbidity $(\sim 30 \%)$ and mortality $(<5 \%)$, absence of right heart failure, absence of extra-hepatic disease, and no peritoneal carcinomatosis (48).

\section{Perioperative management}

Recent studies have highlighted improved perioperative outcomes of patients undergoing liver surgery despite increases in case complexity (49). Nevertheless, several specific considerations should be undertaken for patients with NETs. Specifically, patients with NELM are at risk for carcinoid crisis (6). While some clinicians recommend the use of perioperative octreotide, the appropriate perioperative dosage as well as its actual efficacy remains a matter of debate (50). Hepatic resection is often combined with resection of the primary which appears to be safe (41). For combined operations, liver-directed surgery should typically be performed first in order to take advantage of low central venous pressure. Standard surgical principles of liver surgery should apply for resection of NELM, namely prioritizing low central venous pressure anesthesia, minimizing blood loss, knowledge of liver anatomy, generous use of intraoperative ultrasound, and safe parenchymal transection techniques. Enhanced recovery programs are an important part of complex hepatic surgery programs that hasten recovery and shorten length of hospital stay; these programs should be implemented for patients with NELM (51).

\section{Neoadjuvant and adjuvant treatment strategies}

Following surgery, patients should be followed with cross-sectional imaging and tumor markers every 3-12 months (27). Given the high incidence of recurrence following hepatic resection of NELM $(21,40)$, the use of multimodality therapeutic strategies to improve patient selection for surgery and reduce the development of recurrent disease are appealing. However, little research has been conducted on strategies for preventing recurrence in high-risk NETs. While few studies have investigated the use of adjuvant therapy following resection of NELM (52), some investigators have reported on the use of neoadjuvant therapies to downstage patients in order to facilitate resection of NELM (53). Cloyd et al. reported a single institution retrospective experience of neoadjuvant chemotherapy prior to the resection of pancreatic NELM and reported improved OS compared with immediate surgery, especially among those with synchronous disease (54). Similarly, the orally-available regimen capecitabine and temozolomide has been associated with excellent response rates and long-term outcomes NELM secondary to a primary tumor (55). Additional research is needed to clarify the potential benefits of neoadjuvant and adjuvant therapies for patients with NELM, as well as appropriate selection criteria.

\section{Liver transplantation (LT)}

Given its role in the management of other liver 
Table 1 Indications for LT for NELM

\author{
Milan criteria for LT \\ Biopsy confirmed well-differentiated low-intermediate grade neuroendocrine histology \\ Primary tumor drained by portal system and removed \\ $<50 \%$ involvement of liver \\ Stable/responsive disease for minimum 6 months prior to transplantation \\ Age $<55$ \\ ENETS minimal requirements for LT \\ Well-differentiated low-intermediate grade neuroendocrine histology \\ Primary tumor drained by portal system and removed \\ Absence of extrahepatic disease \\ Expected postoperative mortality $<10 \%$
}

LT, liver transplantation; NELM, neuroendocrine liver metastases; ENETS, European Neuroendocrine Tumor Society.

malignancies, there has long been interest in the use of LT as a potentially curative surgical option for patients with unresectable NELM (56). However, given the shortage of available organs, the frequency of extrahepatic metastatic disease in patients with NETs, and incomplete evidence of its efficacy for NELM, LT has been utilized relatively infrequently for NELM (56). Recent systematic reviews have suggested a 5 -year OS of $>50 \%$, but the data are based on retrospective studies with significant selection biases and inter-study heterogeneity $(1,57)$.

General guidelines for the selection of patients with NELM for LT have been previously outlined (Table 1). After defining criteria for LT for hepatocellular carcinoma, Mazzaferro et al. derived the Milan criteria for NELM, highlighting satisfactory long-term outcomes among these highly selected cases (58). ENETS has similarly identified general guidelines for LT to be used in select indications but note that curative-intent is unlikely and the main indication for LT should be palliation of hormonal syndromes not controlled with other techniques (1). In addition, recent studies have suggested similar survival outcomes among patients within Milan criteria who undergo surgical resection compared to LT (59). Given that good longterm outcomes can be achieved with other liver-directed and systemic therapies for many patients with NELM, and in light of global organ shortages, additional research is needed to define the optimal indications for and outcomes of LT for NELM before it can be incorporated into routine practice.

\section{Liver-directed therapies}

For patients who are not candidates for hepatic resection due to either patient performance status or extent of liver disease, liver-directed therapies are available to assist with locoregional control, improve progression-free survival (PFS), and control hormonal symptoms. Given that the degree of involvement and control of the liver is often the most important predictor of long-term patient outcomes, liver-directed therapies are frequently used for patients with metastatic NET.

\section{Transarterial therapies}

For patients with multifocal bilobar disease, transarterial therapies are usually indicated (60). Transarterial embolization (TAE) can be performed bland (61) or in conjunction with chemotherapy [transarterial chemoembolization (TACE)] (62) or yttrium-90 (63) [transarterial radioembolization (TARE)]. TACE can be performed in a conventional manner utilizing an emulsion of chemotherapy and lipiodol (cTACE) or utilizing chemotherapy drug-eluting beads (DEB-TACE) (64). Patients undergoing TACE frequently experience a postTACE syndrome marked by abdominal pain, nausea, fevers, and transaminitis. While less common, serious complications such as biliary necrosis or abscess as well as mortality can occur (62). TARE results in less severe early toxicity likely because it is less ischemic in nature (63). 
On the other hand, concerns over late toxicity related to radiation fibrosis have been raised particularly given the excellent long-term OS rates observed among these patients (65). Lobar TACE has been associated with a higher incidence of complications among patients who have undergone pancreatoduodenectomy and thus consideration of selective TAE/TACE or TARE as well as periprocedural antibiotics is recommended.

Few studies have directly compared different transarterial treatment strategies. A recent two-institution retrospective analysis noted TACE to have a greater disease control rate, but there was no difference in long-term PFS or OS versus TARE (66). Other studies have suggested that cTACE may lead to improved long-term outcomes compared with TARE, though potentially at the expense of increased early complications (67). A recent three-arm randomized controlled trial comparing TAE, cTACE, and DEB-TACE, was terminated early due to increased severe complications in the DEB-TACE group (68). While decisions regarding liver-directed therapies have traditionally been influenced by institutional preferences, additional prospective comparative studies are needed to define the optimal transarterial therapy.

\section{Other local therapies}

For patients with oligometastatic disease, several modalities exist including percutaneous ablation, external beam radiation, or transarterial therapies. While frequently performed in conjunction with hepatic resection (see above), image-guided ablation can also be performed percutaneously by interventional radiology, typically for patients with recurrent but oligometastatic disease. Percutaneous ablation is most effective in those patients with $1-3$ tumors measuring $<3.5 \mathrm{~cm}$ in diameter. Larger size, hilar location, and proximity to major bile ducts can increase the risk of complications while proximity to large vessels can result in a heat-sink effect limiting its effectiveness. Percutaneous ablation leads to excellent symptom control and good local control but overall recurrence rates are high (69).

External beam radiation is an alternative technique for patients with oligometastatic NELM who are not candidates for hepatic resection. As the effectiveness of ablation decreases as tumor size increases, radiation therapy can be considered for larger solitary tumors $(>3 \mathrm{~cm})$ that are not amenable to resection. Few studies have been conducted on radiation therapy for NELM but external beam radiation using ablative techniques including stereotactic beam radiation therapy (SBRT) and proton beam radiation are frequently used for various primary and secondary liver malignancies $(70,71)$.

\section{Systemic therapies}

In recent years, numerous new systemic therapy options have emerged for patients with well-differentiated GEPNETs $(72,73)$. Often the initial treatment for patients with low-volume well-differentiated NELMs, somatostatin analogs (SSAs) lead to improved PFS and can effectively control the symptoms of hormonal overproduction $(5,74)$.

Advances in the molecular understanding of pancreatic NETs (PNETs) have led to the development of several novel targeted therapies (73). For example, both sunitinib malate, a tyrosine kinase inhibitor, and everolimus, a mammalian target of rapamycin (mTOR) inhibitor, are specifically approved for use in metastatic PNETs $(75,76)$. Everolimus is now also approved for GEP-NETs and pulmonary NETs (77). Emerging research suggests that other antiangiogenic agents, including lenvatinib and cabozantinib, have activity against GEP-NETs (72).

Traditional chemotherapy is also occasionally used for patients with PNETs and less commonly for other types of NETs. While NETs were historically treated with streptozocin-based chemotherapy regimens (78), more recent evidence suggests that the orally available combination capecitabine/temozolomide can lead to significant responses for PNETs (79). Recent evidence also suggests that the proliferation index Ki-67, in addition to providing prognostic information, can also help guide chemotherapy selection. Based on the NORDIC registry, grade 3 well-differentiated NETs with a Ki-67 index of $<55 \%$ may be best treated with capecitabine/temozolomide while tumors with $\mathrm{Ki}-67$ index of $>55 \%$ should be treated with platinum/etoposide which is typically used for poorlydifferentiated neuroendocrine carcinomas $(80,81)$.

An exciting new therapeutic option for patients with metastatic NET, including NELM, is peptide receptor radionuclide therapy (PRRT) that delivers SSA-bound radionuclides selectively to somatostatin receptor positive tumors. PRRT is now approved for use in the United States based on the results of the NETTER-1 trial, which demonstrated improved PFS compared with high-dose SSA among patients with midgut NETs (82). As research into the molecular features of NETs continues, exciting breakthroughs in novel radiopharmaceuticals as well as targeted therapies, checkpoint inhibitors, and oncolytic 


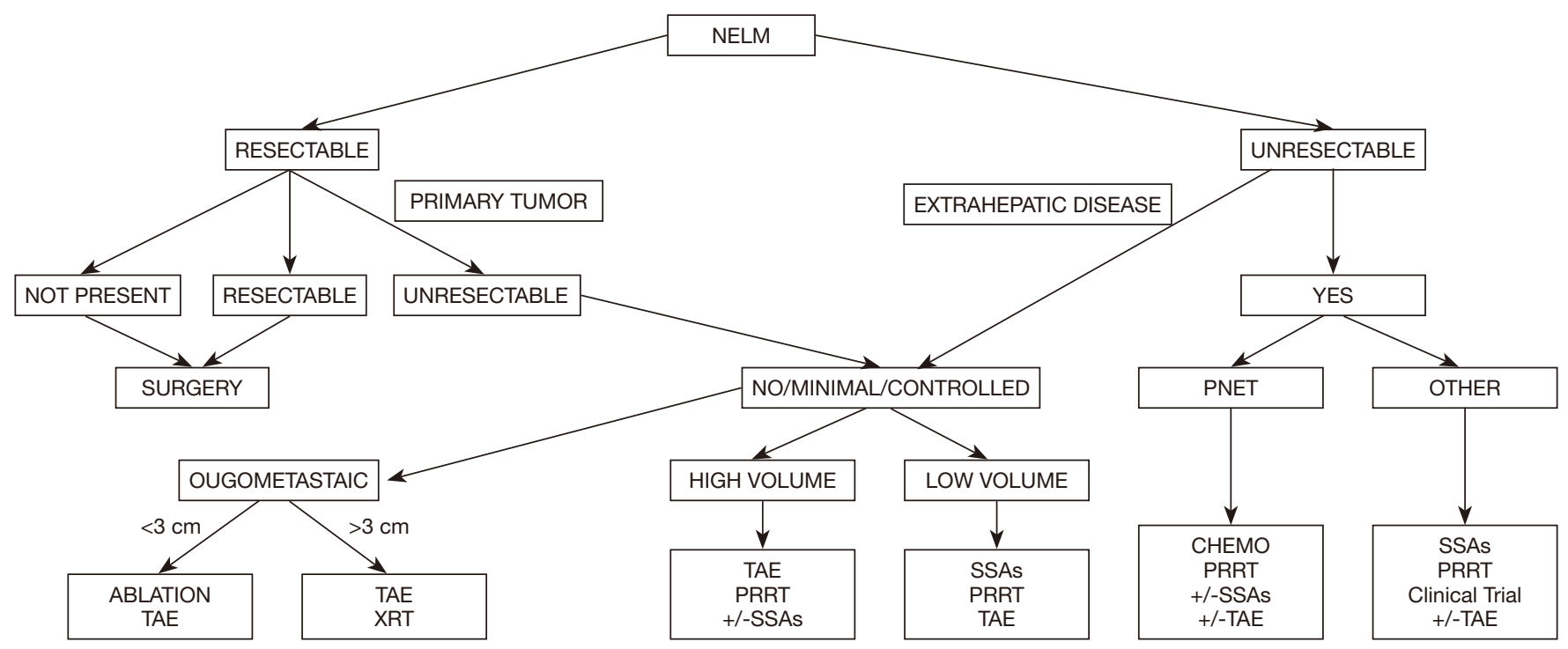

Figure 3 Proposed clinical pathway for managing patients with NELM based on the authors' institutional experience. NELM, neuroendocrine liver metastases; PNET, pancreatic neuroendocrine tumor; TAE, transarterial embolization; XRT, radiation therapy; PRRT; peptide receptor radionuclide therapy; SSAs, somatostatin analogs.

viruses are likely to occur in the near future (72). Additional research into combination therapies such as PRRT with TARE (83), SSAs (84), or chemotherapy (85) are also underway.

\section{Conclusions}

In conclusion, NETs are heterogeneous tumors occurring with increasing frequency that commonly metastasize to the liver. Patients with NELM require a comprehensive evaluation using a multidisciplinary approach. Advances in diagnostic imaging have improved the anatomic staging of patients, commonly identifying multifocal hepatic and extrahepatic metastatic disease. Fortunately, multiple treatment options exist for patients with NELM including surgery, non-surgical liver-directed therapies, and systemic treatment options. Overall, a systematic approach in a multi-disciplinary setting is likely to afford the best longterm outcomes for patients with NELM.

The authors have formulated a proposed algorithm for managing patients with NELM (Figure 3). For patients with resectable tumors, hepatic resection is recommended based on the chance for best long-term outcomes and improved quality of life. Surgical resection may be appropriate even in the setting of low-volume indolent extrahepatic disease or when only $>70 \%$ of liver metastases can be debulked. For patients with unresectable oligometastatic disease, multiple treatment options exist including percutaneous ablation, SBRT, or selective transarterial therapies. For individuals with multifocal bilobar disease, transarterial therapies are prioritized though systemic treatments including PRRT can be considered. For patients with a significant burden of extrahepatic disease, systemic treatments are the recommended option with or without liver-directed therapies depending on the distribution of the liver disease. Nevertheless, high-quality evidence supporting one treatment approach over another as well as for the optimal treatment sequencing is limited. In the near future, ongoing research will be helpful in determining the optimal indications for hepatic surgery, as well as the ideal treatment sequencing for patients with metastatic liver disease.

\section{Acknowledgments}

Funding: None.

\section{Footnote}

Conflicts of Interest: All authors have completed the ICMJE uniform disclosure form (available at http://dx.doi. org/10.21037/hbsn.2020.04.02). TMP serves as an unpaid editorial board member of Hepatobiliary Surgery and 
Nutrition. The other authors have no conflicts of interest to declare.

Ethical Statement: The authors are accountable for all aspects of the work in ensuring that questions related to the accuracy or integrity of any part of the work are appropriately investigated and resolved.

Open Access Statement: This is an Open Access article distributed in accordance with the Creative Commons Attribution-NonCommercial-NoDerivs 4.0 International License (CC BY-NC-ND 4.0), which permits the noncommercial replication and distribution of the article with the strict proviso that no changes or edits are made and the original work is properly cited (including links to both the formal publication through the relevant DOI and the license). See: https://creativecommons.org/licenses/by-nc-nd/4.0/.

\section{References}

1. Pavel M, Baudin E, Couvelard A, et al. ENETS consensus guidelines for the management of patients with liver and other distant metastases from neuroendocrine neoplasms of foregut, midgut, hindgut, and unknown primary. Neuroendocrinology 2012;95:157-76.

2. Yao JC, Hassan M, Phan A, et al. One hundred years after "carcinoid": epidemiology of and prognostic factors for neuroendocrine tumors in 35,825 cases in the United States. J Clin Oncol 2008;26:3063-72.

3. Frilling A, Modlin IM, Kidd M, et al. Recommendations for management of patients with neuroendocrine liver metastases. Lancet Oncol 2014;15:e8-21.

4. Dasari A, Shen C, Halperin D, et al. Trends in the incidence, prevalence, and survival outcomes in patients with neuroendocrine tumors in the United States. JAMA Oncol 2017;3:1335-42.

5. Rinke A, Müller HH, Schade-Brittinger C, et al. Placebocontrolled, double-blind, prospective, randomized study on the effect of octreotide lar in the control of tumor growth in patients with metastatic neuroendocrine midgut tumors: a report from the PROMID study group. J Clin Oncol 2009;27:4656-63.

6. Kimbrough CW, Beal EW, Dillhoff ME, et al. Influence of carcinoid syndrome on the clinical characteristics and outcomes of patients with gastroenteropancreatic neuroendocrine tumors undergoing operative resection. Surgery 2019;165:657-63.

7. Tsilimigras DI, Squires MH, Cloyd JM, et al. Treatment strategies for neuroendocrine liver metastases: an update. Expert Opin Orphan Drugs 2019;7:327-35.

8. Frilling A, Clift AK. Therapeutic strategies for neuroendocrine liver metastases. Cancer. 2015;121:1172-86.

9. Adesoye T, Daleo MA, Loeffler AG, et al. Discordance of histologic grade between primary and metastatic neuroendocrine carcinomas. Ann Surg Oncol 2015;22 Suppl 3:S817-21.

10. Armstrong EA, Beal EW, Shah M, et al. Radiographic characteristics of neuroendocrine liver metastases do not predict clinical outcomes following liver resection. Hepatobiliary Surg Nutr 2020;9:1-12.

11. Dromain C, de Baere T, Baudin E, et al. MR imaging of hepatic metastases caused by neuroendocrine tumors: comparing four techniques. AJR Am J Roentgenol 2003;180:121-8.

12. d'Assignies G, Fina P, Bruno O, et al. High sensitivity of diffusion-weighted MR imaging for the detection of liver metastases from neuroendocrine tumors: comparison with T2-weighted and dynamic gadolinium-enhanced MR imaging. Radiology 2013;268:390-9.

13. Koopmans KP, Neels OC, Kema IP, et al. Improved staging of patients with carcinoid and islet cell tumors with $18 \mathrm{~F}$-dihydroxy-phenyl-alanine and 11C-5-hydroxytryptophan positron emission tomography. J Clin Oncol 2008;26:1489-95.

14. Hofman MS, Kong G, Neels OC, et al. High management impact of Ga-68 DOTATATE (GaTate) PET/CT for imaging neuroendocrine and other somatostatin expressing tumours. J Med Imaging Radiat Oncol 2012;56:40-7.

15. Sadowski SM, Neychev V, Millo C, et al. Prospective study of $68 \mathrm{Ga}$-DOTATATE positron emission tomography/ computed tomography for detecting gastro-enteropancreatic neuroendocrine tumors and unknown primary sites. J Clin Oncol 2016;34:588-96.

16. Hope TA, Bergsland EK, Bozkurt MF, et al. Appropriate use criteria for somatostatin receptor PET imaging in neuroendocrine tumors. J Nucl Med 2018;59:66-74.

17. Ronot M, Clift AK, Baum RP, et al. Morphological and functional imaging for detecting and assessing the resectability of neuroendocrine liver metastases. Neuroendocrinology 2018;106:74-88.

18. Sundin A, Arnold R, Baudin E, et al. ENETS consensus guidelines for the standards of care in neuroendocrine tumors: radiological, nuclear medicine and hybrid imaging. Neuroendocrinology 2017;105:212-44.

19. Gurusamy KS, Ramamoorthy R, Sharma D, et al. Liver 
resection versus other treatments for neuroendocrine tumours in patients with resectable liver metastases. Cochrane Database Syst Rev 2009;(2):CD007060.

20. Fairweather M, Swanson R, Wang J, et al. Management of neuroendocrine tumor liver metastases: long-term outcomes and prognostic factors from a large prospective database. Ann Surg Oncol 2017;24:2319-25.

21. Saxena A, Chua TC, Perera M, et al. Surgical resection of hepatic metastases from neuroendocrine neoplasms: a systematic review. Surg Oncol 2012;21:e131-41.

22. Mayo SC, Pawlik TM. Surgical management of neuroendocrine tumors: treatment of localized and metastatic disease. Oncology (Williston Park) 2011;25:806, 808, 810 .

23. Kaçmaz E, Heidsma CM, Besselink MGH, et al. Treatment of liver metastases from midgut neuroendocrine tumours: a systematic review and meta-analysis. J Clin Med 2019. doi: 10.3390/jcm8030403.

24. Kunz PL, Reidy-Lagunes D, Anthony LB, et al. Consensus guidelines for the management and treatment of neuroendocrine tumors. Pancreas 2013;42:557-77.

25. Singh S, Dey C, Kennecke H, et al. Consensus recommendations for the diagnosis and management of pancreatic neuroendocrine tumors: guidelines from a Canadian National Expert Group. Ann Surg Oncol 2015;22:2685-99.

26. Jin K, Xu J, Chen J, et al. Surgical management for nonfunctional pancreatic neuroendocrine neoplasms with synchronous liver metastasis: a consensus from the Chinese Study Group for Neuroendocrine Tumors (CSNET). Int J Oncol 2016;49:1991-2000.

27. Neuroendocrine and Adrenal Tumors. In: NCCN Clinical Practice Guidelines in Oncology. 2019. Available online: https://www.nccn.org/professionals/physician_gls/default. aspx

28. Spolverato G, Vitale A, Ejaz A, et al. Net health benefit of hepatic resection versus intraarterial therapies for neuroendocrine liver metastases: a Markov decision model. Surgery 2015;158:339-48.

29. Glazer ES, Tseng JF, Al-Refaie W, et al. Long-term survival after surgical management of neuroendocrine hepatic metastases. HPB 2010;12:427-33.

30. Croome KP, Burns JM, G Que F, et al. Hepatic resection for metastatic neuroendocrine cancer in patients with bone metastases. Ann Surg Oncol 2016;23:3693-8.

31. Zhang XF, Beal EW, Weiss M, et al. Timing of disease occurrence and hepatic resection on long-term outcome of patients with neuroendocrine liver metastasis. J Surg
Oncol 2018;117:171-81.

32. Elias D, Lasser P, Ducreux M, et al. Liver resection (and associated extrahepatic resections) for metastatic welldifferentiated endocrine tumors: a 15-year single center prospective study. Surgery 2003;133:375-82.

33. Farley HA, Pommier RF. Surgical treatment of small bowel neuroendocrine tumors. Hematol Oncol Clin North Am 2016;30:49-61.

34. Zhang XF, Beal EW, Chakedis J, et al. Early recurrence of neuroendocrine liver metastasis after curative hepatectomy: risk factors, prognosis, and treatment. J Gastrointest Surg 2017;21:1821-30.

35. Morgan RE, Pommier SJ, Pommier RF. Expanded criteria for debulking of liver metastasis also apply to pancreatic neuroendocrine tumors. Surgery 2018;163:218-25.

36. Ejaz A, Reames BN, Maithel S, et al. Cytoreductive debulking surgery among patients with neuroendocrine liver metastasis: a multi-institutional analysis. HPB 2018;20:277-84.

37. Sarmiento JM, Heywood G, Rubin J, et al. Surgical treatment of neuroendocrine metastases to the liver: a plea for resection to increase survival. J Am Coll Surg 2003;197:29-37.

38. Scott AT, Breheny PJ, Keck KJ, et al. Effective cytoreduction can be achieved in patients with numerous neuroendocrine tumor liver metastases (NETLMs). Surgery 2019;165:166-75.

39. Taner T, Atwell TD, Zhang L, et al. Adjunctive radiofrequency ablation of metastatic neuroendocrine cancer to the liver complements surgical resection. HPB 2013;15:190-5.

40. Mayo SC, de Jong MC, Pulitano C, et al. Surgical management of hepatic neuroendocrine tumor metastasis: results from an international multi-institutional analysis. Ann Surg Oncol 2010;17:3129-36.

41. Scoville S, Xourafas D, Ejaz A, et al. Contemporary indications for and outcomes of hepatic resection for neuroendocrine liver metastases. World J Gastrointest Surg 2020. In Press.

42. Mizuno T, Cloyd JM, Omichi K, et al. Two-stage hepatectomy vs one-stage major hepatectomy with contralateral resection or ablation for advanced bilobar colorectal liver metastases. J Am Coll Surg 2018;226:825-34.

43. Xiang JX, Zhang XF, Weiss M, et al. Early recurrence of well-differentiated (G1) neuroendocrine liver metastasis after curative-intent surgery: risk factors and outcome: XIANG ET AL. J Surg Oncol 2018;118:1096-104. 
44. Minagawa M, Makuuchi M, Takayama T, et al. Selection criteria for repeat hepatectomy in patients with recurrent hepatocellular carcinoma. Ann Surg 2003;238:703-10.

45. Adam R, Bismuth H, Castaing D, et al. Repeat hepatectomy for colorectal liver metastases. Ann Surg 1997;225:51-60; discussion 60-2.

46. Spolverato G, Bagante F, Aldrighetti L, et al. Management and outcomes of patients with recurrent neuroendocrine liver metastasis after curative surgery: An international multi-institutional analysis. J Surg Oncol 2017;116:298-306.

47. Galleberg RB, Knigge U, Tiensuu Janson E, et al. Results after surgical treatment of liver metastases in patients with high-grade gastroenteropancreatic neuroendocrine carcinomas. Eur J Surg Oncol 2017;43:1682-9.

48. O’Toole D, Kianmanesh R, Caplin M. ENETS 2016 consensus guidelines for the management of patients with digestive neuroendocrine tumors: an update. Neuroendocrinology 2016;103:117-8.

49. Cloyd JM, Mizuno T, Kawaguchi Y, et al. Comprehensive complication index validates improved outcomes over time despite increased complexity in 3707 consecutive hepatectomies. Ann Surg 2020;271:724-31.

50. Condron ME, Pommier SJ, Pommier RF. Continuous infusion of octreotide combined with perioperative octreotide bolus does not prevent intraoperative carcinoid crisis. Surgery 2016;159:358-65.

51. Melloul E, Hübner M, Scott M, et al. Guidelines for perioperative care for liver surgery: enhanced recovery after surgery (ERAS) society recommendations. World J Surg 2016;40:2425-40.

52. Chung MH, Pisegna J, Spirt M, et al. Hepatic cytoreduction followed by a novel long-acting somatostatin analog: a paradigm for intractable neuroendocrine tumors metastatic to the liver. Surgery 2001;130:954-62.

53. Perysinakis I, Aggeli C, Kaltsas G, et al. Neoadjuvant therapy for advanced pancreatic neuroendocrine tumors: an emerging treatment modality? Hormones (Athens) 2016;15:15-22.

54. Cloyd JM, Omichi K, Mizuno T, et al. Preoperative fluorouracil, doxorubicin, and streptozocin for the treatment of pancreatic neuroendocrine liver metastases. Ann Surg Oncol 2018;25:1709-15.

55. Squires MH, Worth PJ, Konda B, et al. Neoadjuvant Capecitabine/Temozolomide for Locally Advanced or Metastatic Pancreatic Neuroendocrine Tumors. Pancreas 2020;49:355-60.

56. Clift AK, Frilling A. Liver transplantation and multivisceral transplantation in the management of patients with advanced neuroendocrine tumours. World J Gastroenterol 2018;24:2152-62.

57. Spolverato G, Bagante F, Tsilimigras DI, et al. Liver transplantation in patients with liver metastases from neuroendocrine tumors. Minerva Chir 2019;74:399-406.

58. Mazzaferro V, Pulvirenti A, Coppa J. Neuroendocrine tumors metastatic to the liver: how to select patients for liver transplantation? J Hepatol 2007;47:460-6.

59. Ruzzenente A, Bagante F, Bertuzzo F, et al. Liver resection for neuroendocrine tumor liver metastases within milan criteria for liver transplantation. J Gastrointest Surg 2019;23:93-100.

60. Kennedy A, Bester L, Salem R, et al. Role of hepatic intraarterial therapies in metastatic neuroendocrine tumours (NET): guidelines from the NET-Liver-Metastases Consensus Conference. HPB 2015;17:29-37.

61. Elf AK, Andersson M, Henrikson O, et al.

Radioembolization versus bland embolization for hepatic metastases from small intestinal neuroendocrine tumors: short-term results of a randomized clinical trial. World J Surg 2018;42:506-13.

62. Bloomston M, Al-Saif O, Klemanski D, et al. Hepatic artery chemoembolization in 122 patients with metastatic carcinoid tumor: lessons learned. J Gastrointest Surg 2007;11:264-71.

63. Kennedy AS, Dezarn WA, McNeillie P, et al. Radioembolization for unresectable neuroendocrine hepatic metastases using resin 90Y-microspheres: early results in 148 patients. Am J Clin Oncol 2008;31:271-9.

64. Makary MS, Kapke J, Yildiz V, et al. Conventional versus drug-eluting bead transarterial chemoembolization for neuroendocrine tumor liver metastases. J Vasc Interv Radiol 2016;27:1298-304.

65. Tomozawa Y, Jahangiri Y, Pathak P, et al. Long-term toxicity after transarterial radioembolization with yttrium-90 using resin microspheres for neuroendocrine tumor liver metastases. J Vasc Interv Radiol 2018;29:858-65.

66. Egger ME, Armstrong E, Martin RC 2nd, et al. Transarterial chemoembolization vs radioembolization for neuroendocrine liver metastases: a multi-institutional analysis. J Am Coll Surg 2020;230:363-70.

67. Do Minh D, Chapiro J, Gorodetski B, et al. Intra-arterial therapy of neuroendocrine tumour liver metastases: comparing conventional TACE, drug-eluting beads TACE and yttrium-90 radioembolisation as treatment options using a propensity score analysis model. Eur Radiol 
2017;27:4995-5005.

68. Soulen M, White S, Fidelman N, et al. 03: 27 PM Abstract No. 105 Randomized Embolization Trial for NeuroEndocrine Tumors (RETNET): first safety report. J Vasc Interv Radiol 2019;30:S49-50.

69. Mohan H, Nicholson P, Winter DC, et al. Radiofrequency ablation for neuroendocrine liver metastases: a systematic review. J Vasc Interv Radiol 2015;26:935-42.e1.

70. Ahmed KA, Caudell JJ, El-Haddad G, et al. Radiosensitivity differences between liver metastases based on primary histology suggest implications for clinical outcomes after stereotactic body radiation therapy. Int J Radiat Oncol Biol Phys 2016;95:1399-404.

71. Chuong MD, Kaiser A, Khan F, et al. Consensus report from the miami liver proton therapy conference. Front Oncol 2019;9:457.

72. Cloyd JM, Konda B, Shah MH, et al. The emerging role of targeted therapies for advanced well-differentiated gastroenteropancreatic neuroendocrine tumors. Expert Rev Clin Pharmacol 2019;12:101-8.

73. Scoville SD, Cloyd JM, Pawlik TM. New and emerging systemic therapy options for well-differentiated gastroenteropancreatic neuroendocrine tumors. Expert Opin Pharmacother 2020;21:183-91.

74. Caplin ME, Pavel M, Ćwikła JB, et al. Lanreotide in metastatic enteropancreatic neuroendocrine tumors. N Engl J Med 2014;371:224-33.

75. Raymond E, Dahan L, Raoul JL, et al. Sunitinib malate for the treatment of pancreatic neuroendocrine tumors. N Engl J Med 2011;364:501-13.

76. Yao JC, Shah MH, Ito T, et al. Everolimus for advanced pancreatic neuroendocrine tumors. N Engl J Med 2011;364:514-23.

77. Yao JC, Fazio N, Singh S, et al. Everolimus for the treatment of advanced, non-functional neuroendocrine tumours of the lung or gastrointestinal tract (RADIANT-4): a randomised, placebo-controlled, phase 3 study. Lancet 2016;387:968-77.

Cite this article as: Cloyd JM, Ejaz A, Konda B, Makary MS, Pawlik TM. Neuroendocrine liver metastases: a contemporary review of treatment strategies. HepatoBiliary Surg Nutr 2020;9(4):440-451. doi: 10.21037/hbsn.2020.04.02
78. Moertel CG, Hanley JA, Johnson LA. Streptozocin alone compared with streptozocin plus fluorouracil in the treatment of advanced islet-cell carcinoma. N Engl J Med 1980;303:1189-94.

79. Kunz PL, Catalano PJ, Nimeiri H, et al. A randomized study of temozolomide or temozolomide and capecitabine in patients with advanced pancreatic neuroendocrine tumors: a trial of the ECOG-ACRIN Cancer Research Group (E2211). J Clin Oncol 2018;36:4004.

80. Sorbye H, Welin S, Langer SW, et al. Predictive and prognostic factors for treatment and survival in 305 patients with advanced gastrointestinal neuroendocrine carcinoma (WHO G3): the NORDIC NEC study. Ann Oncol 2013;24:152-60.

81. Milione M, Maisonneuve P, Spada F, et al. The clinicopathologic heterogeneity of grade 3 gastroenteropancreatic neuroendocrine neoplasms: morphological differentiation and proliferation identify different prognostic categories. Neuroendocrinology 2017;104:85-93.

82. Strosberg J, El-Haddad G, Wolin E, et al. Phase 3 trial of 177Lu-Dotatate for midgut neuroendocrine tumors. N Engl J Med 2017;376:125-35.

83. Braat AJAT, Bruijnen RCG, van Rooij R, et al. Additional holmium-166 radioembolisation after lutetium-177dotatate in patients with neuroendocrine tumour liver metastases (HEPAR PLuS): a single-centre, single-arm, open-label, phase 2 study. Lancet Oncol 2020;21:561-70.

84. Yordanova A, Wicharz MM, Mayer K, et al. The role of adding somatostatin analogues to peptide receptor radionuclide therapy as a combination and maintenance therapy. Clin Cancer Res 2018;24:4672-9.

85. Ballal S, Yadav MP, Damle NA, et al. Concomitant $177 \mathrm{Lu}-$ DOTATATE and capecitabine therapy in patients with advanced neuroendocrine tumors: a long-term-outcome, toxicity, survival, and quality-of-life study. Clin Nucl Med 2017;42:e457-66. 